\title{
CORRESPONDENCE
}

\section{Flu: vaccinate to cut risk of chimaeric virus emerging}

SIR - The international scientific community and decision-makers on public health are debating how best to manage the anticipated vaccine shortage for the new pandemic strain of influenza $A$ virus, recently emerged from the animal reservoir. Priority distribution of the first product batches must be to individuals at high risk and to crucial employees.

But decisions about priority distribution should also take into account the benefits to the international community of vaccination in developing countries. Viral 'shedding' (the expulsion of virus) is reduced in vaccinated individuals and therefore the risk of reassortment with animal viruses is decreased. This consideration is in line with the 'One Health' vision - a multidisciplinary initiative to improve the health of humans, animals and the environment that is endorsed by the United Nations Food and Agriculture Organization, the World Health Organization and the World Organisation for Animal Health (OIE).

There is a risk of generating novel influenza A viruses through reassortment of the eight genes that result in antigenic shift, which would give rise to strains against which the human population has no immunity. For example, it was reassortment between avian and human influenza viruses that created the human pandemic viruses of 1957 and 1968 (K. Subbarao et al. in Influenza Virology Current Topics ed. Y. Kawaoka, 229-280, Caister Academic; 2006).

We are at present in a unique situation with the worldwide spread of the latest pandemic H1N1 virus, known as novel animal-origin H1N1 (naoH1N1) virus. Concurrently, and possibly for the first time in history, several developing countries are experiencing widespread infections in poultry by avian influenza viruses of the $\mathrm{H} 5 \mathrm{~N} 1$ and H9N2 subtypes, which also infect humans. In addition, H5N1 viruses that are widespread in Africa, the Middle East and Asia contain genetic mutations that reflect increased virulence for humans (see, for example, E. De Wit and R. A. M. Fouchier J. Clin. Virol. 41, 1-6; 2008, and G. Cattoli et al. PLOS ONE 4, e4842; 2009).

In countries where animal husbandry practices fall short of accepted biosecurity standards and where immunologically naive animal caretakers infected with naoH1N1 would shed large amounts of infectious virus, there is a significant risk of emergence of a reassortant virus. A reassortant virus containing a combination of genes, including a novel human-adapted influenza virus and $\mathrm{H} 5 \mathrm{~N} 1$ or $\mathrm{H} 9 \mathrm{~N} 2$, could result in chimaeric viruses with unknown characteristics.

Fast-tracking vaccination of humans against pandemic influenza in developing countries where flu in poultry is endemic would help prevent reassortment between naoH1N1 or other novel pandemic influenza strains and avian influenza viruses. That would deflect the unpredictable and serious consequences of viral reassortment to humankind worldwide.

Ilaria Capua, Giovanni Cattoli OIE Collaborating Centre for Diseases at the Human-Animal Interface, Istituto Zooprofilattico Sperimentale delle Venezie, 35020 Legnaro, Padova, Italy e-mail: icapua@izsvenezie.it

\section{Flu: weighing up conflicting expert information}

SIR - In his Essay 'Pandemics: avoiding the mistakes of 1918' (Nature 459, 324-325; 2009), John M. Barry writes that during an influenza pandemic "telling the public the truth is ... paramount". Truth telling is, he notes, the basis for trust and compliance in public-health measures. However, the H1N1 influenza debate in the Netherlands illustrates that things may not be that simple.

In the current 'swine flu' pandemic, the expert sources of information and guidance for the Dutch public are Albert Osterhaus of the National Influenza Centre in Rotterdam and Roel Coutinho at the National Institute for Public Health and the Environment in Bilthoven. These two experts are not strangers to such challenges: both have often been consulted on risks to the public since the 1980s.

Since the early years of the pandemic debate, Osterhaus has kept us informed about the greater picture, warning us of an 'imminent' flu pandemic (see, for example, J. C. de Jong et al. Nature 389, 554; 1997, and the Dutch newspaper De Volkskrant, 2 May 2009). Coutinho, by contrast, has stuck to the immediate facts (see, for example, the newspaper NRC Handelsblad, 14 March 2006), and cautioned us that "there's no need to panic" (see, for example, the newspaper Het Parool, 1 May 2009). In communicating their different perspectives, these virologists were conveying conflicting messages to the general public, even though their aims were the same - to protect the public's health.

Public understanding, therefore, cannot hinge only on experts telling the truth as they see it: also important is how the public interprets mixed messages. It is the public's ability to assess the relative value of expert information that helps to stimulate the trust and compliance needed to follow recommended public-health measures.

That trust will be particularly crucial during the months to come, in implementing the planned vaccination of the entire Dutch population - and the related information campaign following the announcement made by the World Health Organization on 11 June of a pandemic, which is expected to strike the Netherlands with

force this autumn.

Erwin van Rijswoud Institute for Science, Innovation and Society, Radboud University Nijmegen, $6500 \mathrm{GL}$ Nijmegen, the Netherlands e-mail: e.vanrijswoud@science.ru.nl

\section{Where will we find the tritium to fuel hybrid reactors?}

SIR - In your News Feature 'The hybrid returns' (Nature 460, 25-28; 2009), you discuss the feasibility of creating a fusionfission hybrid reactor to generate greenhouse-gas-free and wastefree nuclear energy. However, there is another challenge to add to the factors to be considered: where would the hundreds of grams of tritium needed daily to fuel the deuterium-tritium fusion reaction be produced?

For every neutron produced in this reaction, and in theory destined to burn the radioactive waste from the fission reaction, a tritium atom must be bred in a lithium (or lithium/lead) reactor blanket. The conventional, purefusion design demands a breeding ratio that is greater than one, to allow for losses and the decay of tritium; this might be extremely difficult to achieve even with essential neutron multipliers such as beryllium. The significant fraction of neutrons required to treat fissile material means that there could not possibly be enough to breed tritium as well.

At present, gram-scale quantities are available from Canadian sources, but the net production rate is only about 500 grams per year - a trivial amount compared with fusion-reactor requirements. For example, starting up any type of fusion reactor will need several months' supply of tritium just to bring the breeding process on stream. This issue is better addressed now than in 30 to 50 years' time.

\section{J. H. Evans Abingdon,}

Oxon OX14 2EQ, UK

e-mail:jhevans@sky.com 